\title{
Transient elastic waves propagating in a multi-layered medium subjected to in-plane dynamic loadings. I. Theory
}

\author{
By G.-S. LEE A D C.-C. M A \\ Department of Mechanical Engineering, National Taiwan University, \\ Taipei, Taiwan 10617, Republic of China
}

Received 11 February 1999; revised 5 August 1999; accepted 20 September 1999

The propagation of elastic transient waves in a multi-layered medium subjected to in-plane loadings is investigated in this study. One of the objectives of this study is to develop an effective analytical method for determining transient full-field solutions in the layered medium. A matrix method is developed by expanding the matrix solution obtained directly from the boundary-value problem in the integral transform domain into a power series of the phase-related reflection and transmission matrix which characterizes the multiple reflections and transmissions of all waves in every layer. The transient response of the multi-layered medium is decomposed into infinite wave groups in which the waves are either reflected by, or transmitted through, the interfaces. The connection between the proposed matrix method and the generalized ray method is established for the layered medium in the transform domain. The matrix representation of the solution enables us to calculate the transient response of the layered medium without tracing the ray path manually. The obtained analytical solution can easily be applied to numerical calculations. The double inverse transform is performed based on Cagniard's method and the theoretical transient solution for a layered half-space subjected to an in-plane dynamic force will be presented in part II. An experimental set-up that simulates the plane stress condition for a layered half-space was established to obtain the dynamic displacement response. The experimental result agrees well with the theoretical solution. The proposed methodology in this study can be extended to solve more complicated problems such as waves propagating in three-dimensional space.

Keywords: multi-layered medium; wave propagation;

transient analysis; dynamic loadings

\section{Introduction}

Wave propagation in layered media has been studied extensively because of its wide technological applications. One of the basic models in theoretical seismology is that of $N$ homogeneous layers welded on top of a half-space. A large amount of the study on modern seismology focuses on calculating the response of such a medium to sudden disturbances due to sources located either on the surface or inside the medium. In a deterministic formulation, it possesses a developed and rather systematic methodology. Thomson (1950) and Haskell (1953) proposed a transfer matrix method to 
determine the dispersion relation for the propagation of seismic waves within the Earth modelled by a number of uniform layers. In the Thomson-Haskell matrix formulation, the displacement-traction vector at the top surface of a layer is related to that at the bottom surface by a transfer matrix, and thus is carried cross the interface continuously through the entire stack by the product of transfer matrices. The unknown variables in the displacement-traction vector at each layer are then determined by reformulating the matrix product to satisfy the boundary conditions at both ends. Gilbert \& Backus (1966) described a general method, the propagator technique, that systematized the transfer matrix method for a general stratification in elastic parameters. Similar to the transfer matrix method, the reflectivity method was developed by Fuchs (1968) and Fuchs \& Müller (1971). The reflection and transmission of plane waves at layered media were treated first, followed by the synthesis of point source wave fields, and the theoretical seismograms were calculated by recursive methods. Based on the propagator technique and reflectivity method, Kennett \& Kerry (1979) proposed a reflection matrix method, which can be used to construct the entire response in terms of reflection and transmission matrices, in analysing the excitation induced by general sources in a stratified medium. The reflectivity method was extended later by Müller (1985). A unified framework was presented by Kennett (1983) for all classes of seismic phenomena in stratified media. On the other hand, Ma \& Huang (1996) derived the transfer relation as the general representations of the responses between each layer, instead of the displacement-traction vector, to determine the transient wave propagating in a layered medium. A stochastic formulation of the problem better describes physical reality, but on the other hand complicates mathematical treatment of particular tasks. In the literature there exist a number of papers (see Ziegler 1977; Scott 1985) where some problems of stochastic wave propagation in layered elastic media are analysed.

Propagation of elastic or viscoelastic harmonic waves through a stratified medium was investigated by Kotulski (1990) and Caviglia \& Morro (1994). The modelling has been made more realistic by letting the material be anisotropic (Nayfeh 1991, 1995) or porous (Lauriks et al. 1991). The transition matrix formulation of acoustic scattering from an arbitrary number of scatterers was derived by Peterson \& Ström (1974). The resulting total transition matrix was expressed in terms of the individual transition matrices and in terms of functions which describe the configuration of the scatterers.

In determining the transient waves in a plate, the methods that start with a given boundary-value problem were proposed by Mencher (1953), Knopoff (1958) and Davids (1959) with the application of the Bromwich expansion. The transient wave solution for a two-layered solid (a layer overlaying a half-space) was obtained by Pekeris et al. (1965). The solutions were first expressed in an algebraic form and then expanded into a series, with each term representing a wave propagating in the medium. The expansion requires the evaluation of a $4 \times 4$ determinant for the plate and a $6 \times 6$ determinant for the two-layered medium. Since the complexity of solutions increases as the number of boundary conditions rises, the direct Bromwich expansion becomes complicated in the application of the ray theory to media with many layers.

Spencer (1960) proposed the method of generalized rays to obviate the necessity for solving the tedious boundary-value problem by constructing the solution from the application of the results of simpler problems. In his approach, the Laplace- 
transformed solution of two canonical problems involving the reflection and transmission of arbitrary source incident waves at a single interface was considered. The results of these canonical problems were then combined to form an individual generalized ray which is an integral representation of waves along a prescribed ray path. The method leads to an infinite series of the generalized ray integral constructed in the Laplace transform domain by assembling the source function, reflection and transmission coefficient, the receiver function and the phase function. The inverse of the ray integral was found in a closed form by applying the Cagniard method (Cagniard 1939). Since the transient response for the layered medium is exact up to the arrival time of the next ray, only a finite number of rays will be involved in the early-time solution. The theory of generalized rays was cast to determine the transient response of a plate by Ceranoglu \& Pao (1981), of a multi-layered medium by Müller $(1968 a, b, 1969)$, and the theory was reviewed by Pao \& Gajewski (1977). For the long-time response at a receiver in the layered medium, however, the difficulty arises in determining a large number of generalized ray paths along which the rays arrived at the receiver.

In this paper the authors will show how the generalized ray solutions for a multilayered medium can be constructed directly by solving the corresponding boundaryvalue problems, with the aid of the integral transform technique and the matrix Bromwich expansion. The wave field for each layer of the multi-layered medium will be represented in the double Laplace transform domain by two kinds of field vector, one for the waves propagating in the direction of increasing $y$ and the other in the direction of decreasing $y$. The phase-related receiver matrix for each layer that relates the field vector to the response functions (displacement, stress, etc.) was obtained. The interface and boundary conditions were applied to obtain the system of equations for determining the global field vector that is a stack of the field vectors in each layer. The global field vector is thus expressed in a closed matrix form, instead of the algebraic form in Pekeris's solution. By rearranging the coefficient matrix in a special form consisting of the diagonal, lower-triangle and upper-triangle parts, and extracting the diagonal part from it, the matrix Bromwich expansion was applied to obtain the global phase-related reflection and transmission matrix which characterizes the multiple reflections and transmissions of waves in every layer. After the application of the matrix Bromwich expansion, the solution in the transform domain will be obtained in an infinite matrix series form given by assembling the phaserelated receiver matrix, reflection and transmission matrix and source vector. This approach leads itself to ray interpretation. The transient response can be obtained by the application of Cagniard's method for Laplace inversion. With aids of the proposed method, the phase function that is determined manually in the theory of generalized ray can be determined automatically by the phase-related matrices and vectors. Furthermore, the matrix formulation presented in this study is particularly suitable for the numerical computation.

\section{Statement of the problem}

Consider an initially undisturbed multi-layered medium consisting of $n$ layers separated by parallel planes, as depicted in figure 1. Each layer is assumed homogeneous and isotropic, and the discontinuity condition is considered at the interfaces. All quantities related to a specified $i$ th layer are suffixed by a superscript or subscript $i$

Proc. R. Soc. Lond. A (2000) 
in parentheses. For plane strain problems, the Cartesian coordinate system is chosen so that the response is a function of $(x, y, t)$. Uniformly distributed line loadings parallel to the $z$-axis are applied either at interfaces or within layers. Since responses for the medium subjected to dynamic loadings which are located within layers can be obtained by introducing artificial interfaces at the applied-loading locations, we consider all applied loadings that are located at interfacial or lateral surfaces of the multi-layered medium. The boundary conditions on the top and bottom layers of the medium can be written as

$$
\left.\begin{array}{rl}
\sigma_{y y}^{(1)}(x, 0, t) & =\sigma_{y y}^{[0]}(x, t), \\
\sigma_{x y}^{(1)}(x, 0, t) & =\sigma_{x y}^{[0]}(x, t), \\
\sigma_{y y}^{(n)}\left(x,-h_{n}, t\right) & =\sigma_{y y}^{[n]}(x, t), \\
\sigma_{x y}^{(n)}\left(x,-h_{n}, t\right) & =\sigma_{x y}^{[n]}(x, t),
\end{array}\right\}
$$

for $-\infty<x<\infty$, where

$$
h_{n}=\sum_{i=1}^{n} h^{(i)},
$$

in which $h^{(i)}$ is the thickness of the $i$ th layer. Loadings applied at the interface $y=-h_{i}$ between two adjacent layers yield the traction and displacement discontinuity conditions

$$
\left.\begin{array}{r}
u^{(i)}\left(x,-h_{i}, t\right)-u^{(i+1)}\left(x,-h_{i}, t\right)=u^{[i]}(x, t), \\
v^{(i)}\left(x,-h_{i}, t\right)-v^{(i+1)}\left(x,-h_{i}, t\right)=v^{[i]}(x, t), \\
\sigma_{y y}^{(i)}\left(x,-h_{i}, t\right)-\sigma_{y y}^{(i+1)}\left(x,-h_{i}, t\right)=\sigma_{y y}^{[i]}(x, t), \\
\sigma_{x y}^{(i)}\left(x,-h_{i}, t\right)-\sigma_{x y}^{(i+1)}\left(x,-h_{i}, t\right)=\sigma_{x y}^{[i]}(x, t),
\end{array}\right\}
$$

$i=1,2,3, \ldots, n-1$, where the superscripts $i$ in parentheses (i.e. . $(i)$ and . $\left.^{(i+1)}\right)$ denote the field quantities in the $i$ th layer and the $(i+1)$ th layer, respectively. Furthermore, in equations (2.1) and (2.2), the applied loading at the interface $i$ is distinguished from the field quantities by attaching a superscript $i$ in a bracket (i.e. . ${ }^{[i]}$ ).

To simplify the expression, an applied displacement-traction vector $\boldsymbol{t}^{[i]}$ (for $i=0,1, \ldots, n)$ at the $n+1$ planes is defined,

$$
\boldsymbol{t}^{[0]}=\left(\begin{array}{ll}
\sigma_{y y}^{[0]} & \sigma_{x y}^{[0]}
\end{array}\right), \quad \boldsymbol{t}^{[n]}=\left(\begin{array}{ll}
\sigma_{y y}^{[n]} & \sigma_{x y}^{[n]}
\end{array}\right)
$$

and

$$
\boldsymbol{t}^{[i]}=\left(\begin{array}{llll}
u^{[i]} & v^{[i]} & \sigma_{y y}^{[i]} & \sigma_{x y}^{[i]}
\end{array}\right)^{\mathrm{T}} \quad \text { for } i=1,2, \ldots, n-1 .
$$

If there is no discontinuity at the interface $i$, the applied displacement-traction vector at this interface will vanish, i.e.

$$
\boldsymbol{t}^{[i]}=\left(\begin{array}{llll}
0 & 0 & 0 & 0
\end{array}\right)^{\mathrm{T}} .
$$

When a concentrated vertical force is applied at the interface, the discontinuity is given by

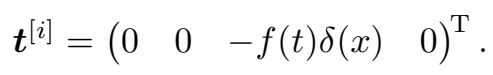




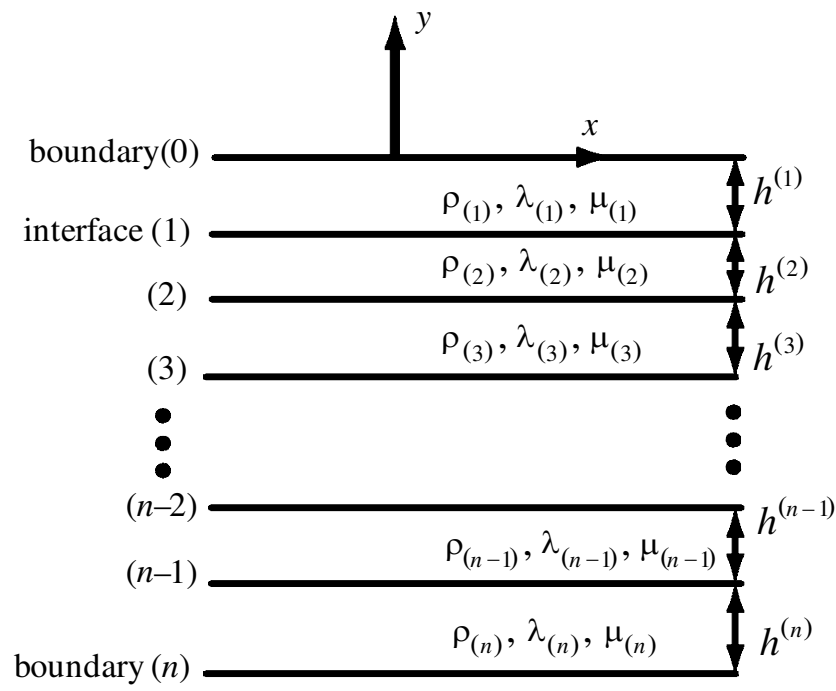

Figure 1. Configuration and coordinate system of an $n$-layered medium.

The discontinuity caused by a concentrated horizontal force is then

$$
\boldsymbol{t}^{[i]}=\left(\begin{array}{llll}
0 & 0 & 0 & -f(t) \delta(x)
\end{array}\right)^{\mathrm{T}} .
$$

For a glide edge dislocation or a horizontal slip fault suddenly generated at time $t=0$ along the interface $i$, the problem can be represented by assuming (Boore et al. 1971)

$$
\boldsymbol{t}^{[i]}=(-b H(t) H(x) \quad 0 \quad 00 \quad 0)^{\mathrm{T}},
$$

where $b$ denotes the magnitude of Burger's vector. The solutions for various types of dynamic loadings can be obtained by a linear superposition.

\section{Formulations in double transform domain}

In absence of body forces, the two-dimensional equations of motion expressed in terms of two displacement potentials $\phi$ and $\psi$ are (Achenbach 1973)

$$
\begin{aligned}
& \frac{\partial^{2} \phi}{\partial x^{2}}+\frac{\partial^{2} \phi}{\partial y^{2}}=s_{\mathrm{L}}^{2} \frac{\partial^{2} \phi}{\partial t^{2}}, \\
& \frac{\partial^{2} \psi}{\partial x^{2}}+\frac{\partial^{2} \psi}{\partial y^{2}}=s_{\mathrm{T}}^{2} \frac{\partial^{2} \psi}{\partial t^{2}},
\end{aligned}
$$

where

$$
s_{\mathrm{L}}=\sqrt{\frac{\rho}{\lambda+2 \mu}}=\frac{1}{c_{\mathrm{L}}}, \quad s_{\mathrm{T}}=\sqrt{\frac{\rho}{\mu}}=\frac{1}{c_{\mathrm{T}}} .
$$

Here $\rho$ is the mass density of the material, $\lambda$ and $\mu$ are elastic constants of Lamé, and $s_{\mathrm{L}}$ and $s_{\mathrm{T}}$ are slownesses of longitudinal and transverse waves, respectively. 
Displacement components are given in terms of the two potentials by

$$
\begin{aligned}
& u=\frac{\partial \phi}{\partial x}+\frac{\partial \psi}{\partial y} \\
& v=\frac{\partial \phi}{\partial y}-\frac{\partial \psi}{\partial x}
\end{aligned}
$$

where $u$ and $v$ are the displacements in the $x$ - and $y$-directions, respectively. On invoking Hooke's law and equation (3.2), the stress components are

$$
\left.\begin{array}{l}
\sigma_{x x}=\mu\left(\left(s_{\mathrm{T}}^{2}-2 s_{\mathrm{L}}^{2}\right) \frac{\partial^{2} \phi}{\partial t^{2}}+2\left(\frac{\partial^{2} \phi}{\partial x^{2}}+\frac{\partial^{2} \psi}{\partial x \partial y}\right)\right), \\
\sigma_{y y}=\mu\left(\left(s_{\mathrm{T}}^{2}-2 s_{\mathrm{L}}^{2}\right) \frac{\partial^{2} \phi}{\partial t^{2}}+2\left(\frac{\partial^{2} \phi}{\partial y^{2}}-\frac{\partial^{2} \psi}{\partial x \partial y}\right)\right), \\
\sigma_{x y}=\mu\left(2 \frac{\partial^{2} \phi}{\partial x \partial y}+\frac{\partial^{2} \psi}{\partial y^{2}}-\frac{\partial^{2} \psi}{\partial x^{2}}\right) .
\end{array}\right\}
$$

We will seek expressions for the field variables by applying the one-sided Laplace transform over time $t$ and the two-sided Laplace transform over spatial coordinate $x$. The definition of a function $f(x, y, t)$ in the double Laplace transform domain is given by

$$
\hat{f}(y ; \xi, p)=\int_{-\infty}^{\infty} \mathrm{e}^{-p \xi x} \int_{0}^{\infty} f(x, y, t) \mathrm{e}^{-p t} \mathrm{~d} t \mathrm{~d} x,
$$

where $p$ is a positive real number, large enough to ensure the convergence of the integral, and $\xi$ is a complex variable. The inverse formulation is given by

$$
f(x, y, t)=\frac{-p}{4 \pi^{2}} \int_{\xi_{1}-\infty \mathrm{i}}^{\xi_{1}+\infty \mathrm{i}} \mathrm{e}^{p \xi x} \int_{p_{1}-\infty \mathrm{i}}^{p_{1}+\infty \mathrm{i}} \hat{f}(y ; \xi, p) \mathrm{e}^{p t} \mathrm{~d} p \mathrm{~d} \xi
$$

By applying the double Laplace transform according to the definition given by $(3.4 a)$, equations $(3.1 a)$ and $(3.1 b)$ become two ordinary differential equations with the following general solutions,

$$
\begin{aligned}
\hat{\phi}(y ; \xi, p) & =\phi_{-}(\xi, p) \mathrm{e}^{+p \gamma_{\mathrm{L}} y}+\phi_{+}(\xi, p) \mathrm{e}^{-p \gamma_{\mathrm{L}} y} \\
\hat{\psi}(y ; \xi, p) & =\psi_{-}(\xi, p) \mathrm{e}^{+p \gamma_{\mathrm{T}} y}+\psi_{+}(\xi, p) \mathrm{e}^{-p \gamma_{\mathrm{T}} y}
\end{aligned}
$$

where

$$
\gamma_{\mathrm{L}}=\sqrt{s_{\mathrm{L}}^{2}-\xi^{2}} \text { and } \gamma_{\mathrm{T}}=\sqrt{s_{\mathrm{T}}^{2}-\xi^{2}}
$$

The condition $\operatorname{Re} \gamma_{\mathrm{L}} \geqslant 0\left(\operatorname{Re} \gamma_{\mathrm{T}} \geqslant 0\right)$ is satisfied by providing branch cuts along $s_{\mathrm{L}} \leqslant|\operatorname{Re} \xi|<\infty\left(s_{\mathrm{T}} \leqslant|\operatorname{Re} \xi|<\infty\right), \operatorname{Im} \xi=0$, and choosing the branch of positive square roots. The unknown functions $\phi_{-}, \psi_{-}, \phi_{+}$and $\psi_{+}$are four field coefficients of each layer, to be determined by boundary conditions. The field coefficients expressed in $(3.5 a)$ and $(3.5 b)$ with subscript ' - ' denote the waves propagating in the direction of decreasing $y$ (downgoing waves) and those with subscript ' + ' are the waves propagating in the direction of increasing $y$ (upgoing waves). 
For convenience, the field coefficients in (3.5) are arranged into two column matrices,

$$
\boldsymbol{c}_{-}=\left(\begin{array}{ll}
\phi_{-} & \psi_{-}
\end{array}\right)^{\mathrm{T}}
$$

and

$$
\boldsymbol{c}_{+}=\left(\phi_{+} \psi_{+}\right)^{\mathrm{T}}
$$

for downgoing and upgoing waves, respectively. From (3.2) and (3.3), and introducing the displacement vector $\boldsymbol{u}=\left(\begin{array}{ll}\hat{u} & \hat{v}\end{array}\right)^{\mathrm{T}}$ and traction vector $\boldsymbol{f}=\left(\begin{array}{ll}\hat{\sigma}_{y y} & \hat{\sigma}_{x y}\end{array}\right)^{\mathrm{T}}$, the displacement-traction vector and field coefficient matrices in transform domain are related as follows,

$$
\left(\begin{array}{c|c}
\boldsymbol{u}(y) \\
\hline \boldsymbol{f}(y)
\end{array}\right)=\left(\begin{array}{ll}
\boldsymbol{M}_{11}(y) & \boldsymbol{M}_{12}(y) \\
\hline \boldsymbol{M}_{21}(y) & \boldsymbol{M}_{22}(y)
\end{array}\right)\left(\begin{array}{c}
\boldsymbol{c}_{-} \\
\hline \boldsymbol{c}_{+}
\end{array}\right),
$$

where

$$
\begin{aligned}
& \boldsymbol{M}_{11}(y)=p\left[\begin{array}{cc}
\xi \mathrm{e}^{-p \gamma_{\mathrm{L}} y} & \gamma_{\mathrm{T}} \mathrm{e}^{-p \gamma_{\mathrm{T}} y} \\
\gamma_{\mathrm{L}} \mathrm{e}^{-p \gamma_{\mathrm{L}} y} & -\xi \mathrm{e}^{-p \gamma_{\mathrm{T}} y}
\end{array}\right], \\
& \boldsymbol{M}_{12}(y)=p\left[\begin{array}{cc}
\xi \mathrm{e}^{p \gamma_{\mathrm{L}} y} & -\gamma_{\mathrm{T}} \mathrm{e}^{p \gamma_{\mathrm{T}} y} \\
-\gamma_{\mathrm{L}} \mathrm{e}^{p \gamma_{\mathrm{L}} y} & -\xi \mathrm{e}^{p \gamma_{\mathrm{T}} y}
\end{array}\right], \\
& \boldsymbol{M}_{21}(y)=\mu p^{2}\left[\begin{array}{cc}
\left(s_{\mathrm{T}}^{2}-2 \xi^{2}\right) \mathrm{e}^{-p \gamma_{\mathrm{L}} y} & -2 \xi \gamma_{\mathrm{T}} \mathrm{e}^{-p \gamma_{\mathrm{T}} y} \\
2 \xi \gamma_{\mathrm{L}} \mathrm{e}^{-p \gamma_{\mathrm{L}} y} & \left(s_{\mathrm{T}}^{2}-2 \xi^{2}\right) \mathrm{e}^{-p \gamma_{\mathrm{T}} y}
\end{array}\right], \\
& \boldsymbol{M}_{22}(y)=\mu p^{2}\left[\begin{array}{cc}
\left(s_{\mathrm{T}}^{2}-2 \xi^{2}\right) \mathrm{e}^{p \gamma_{\mathrm{L}} y} & +2 \xi \gamma_{\mathrm{T}} \mathrm{e}^{p \gamma_{\mathrm{T}} y} \\
-2 \xi \gamma_{\mathrm{L}} \mathrm{e}^{p \gamma_{\mathrm{L}} y} & \left(s_{\mathrm{T}}^{2}-2 \xi^{2}\right) \mathrm{e}^{p \gamma_{\mathrm{T}} y}
\end{array}\right] .
\end{aligned}
$$

Response functions, such as displacement and stress components, can be obtained by multiplying each of the four field coefficients with suitable transfer functions in double transform domain and then adding each of their contributions. The phase-related receiver matrices $\boldsymbol{M}_{11}(y), \boldsymbol{M}_{12}(y), \boldsymbol{M}_{21}(y)$ and $\boldsymbol{M}_{22}(y)$ facilitate the expression of response functions.

\section{Waves propagating in a multi-layered medium}

On invoking boundary and discontinuity conditions in (2.1) and (2.2) and applying the double Laplace transform, a system of equations are developed to determine the field matrices $\boldsymbol{c}_{-}^{(i)}$ and $\boldsymbol{c}_{+}^{(i)}$ for each layer as follows,

$$
\left.\begin{array}{r}
\left(\begin{array}{cc}
M_{21}^{(1)}(0) & -M_{22}^{(1)}(0)
\end{array}\right)\left(\begin{array}{c}
\boldsymbol{c}_{-}^{(1)} \\
\boldsymbol{c}_{+}^{(1)}
\end{array}\right)=\hat{\boldsymbol{t}}^{[0]} \quad \text { at top surface } y=0, \\
\left(\begin{array}{ll}
\boldsymbol{M}_{21}^{(n)}\left(-h_{n}\right) & -\boldsymbol{M}_{22}^{(n)}\left(-h_{n}\right)
\end{array}\right)\left(\begin{array}{c}
\boldsymbol{c}_{-}^{(n)} \\
\boldsymbol{c}_{+}^{(n)}
\end{array}\right)=\hat{\boldsymbol{t}}^{[n]} \quad \text { at bottom surface } y=-h_{n},
\end{array}\right\}
$$




$$
\left.\begin{array}{r}
\left(\begin{array}{llll}
\boldsymbol{M}_{11}^{(i)}\left(-h_{i}\right) & \boldsymbol{M}_{12}^{(i)}\left(-h_{i}\right) & -\boldsymbol{M}_{11}^{(i+1)}\left(-h_{i}\right) & -\boldsymbol{M}_{12}^{(i+1)}\left(-h_{i}\right) \\
\boldsymbol{M}_{21}^{(i)}\left(-h_{i}\right) & \boldsymbol{M}_{22}^{(i)}\left(-h_{i}\right) & -\boldsymbol{M}_{21}^{(i+1)}\left(-h_{i}\right) & -\boldsymbol{M}_{22}^{(i+1)}\left(-h_{i}\right)
\end{array}\right)\left(\begin{array}{c}
\boldsymbol{c}_{-}^{(i)} \\
\boldsymbol{c}_{+}^{(i)} \\
\boldsymbol{c}_{-}^{(i+1)} \\
\boldsymbol{c}_{+}^{(i+1)}
\end{array}\right)=\hat{\boldsymbol{t}}^{[i]} \\
\text { at interface } y=-h_{i}, \quad i=1,2, \ldots, n-1, n
\end{array}\right\}
$$

where $\hat{\boldsymbol{t}}^{[i]}$ indicates the transformed applied displacement-traction vector $\boldsymbol{t}^{[i]}$ as defined in (2.3). Introducing a global field vector $\boldsymbol{c}$ ( $4 n$ elements) for the multilayered medium,

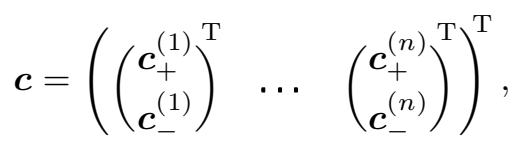

and the global boundary displacement-traction vector $\hat{\boldsymbol{t}}$ ( $4 n$ elements), which is stacked by the applied loadings $\hat{\boldsymbol{t}}^{[i]}$ at all interfaces and boundaries, can be expressed by

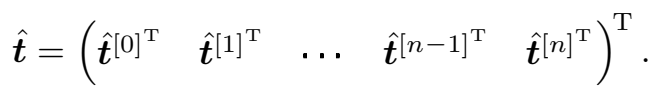

Equation (4.1) can be rewritten in the transformed domain in a more compact form as

$$
M c=\hat{t}
$$

where the coefficient matrix $\boldsymbol{M}$ is a $4 n \times 4 n$ matrix with bandwidth 11 given by

$$
\boldsymbol{M}=\boldsymbol{D}+\boldsymbol{L}+\boldsymbol{U}=\left[\begin{array}{ccccccc}
\boldsymbol{D}_{0} & \boldsymbol{U}_{0} & & & & & \\
\boldsymbol{L}_{1} & \boldsymbol{D}_{1} & \boldsymbol{U}_{1} & & & & \\
& \boldsymbol{L}_{2} & \boldsymbol{D}_{2} & \ddots & & & \\
& & \ddots & \ddots & \ddots & & \\
& & \ddots & \boldsymbol{D}_{n-2} & \boldsymbol{U}_{n-2} & \\
& & & & \boldsymbol{L}_{n-1} & \boldsymbol{D}_{n-1} & \boldsymbol{U}_{n-1} \\
& & & & & \boldsymbol{L}_{n} & \boldsymbol{D}_{n}
\end{array}\right]
$$

In (4.5), the components of the diagonal matrix $\boldsymbol{D}$ are given by

$$
\left.\begin{array}{rl}
\boldsymbol{D}_{0} & =\boldsymbol{M}_{21}^{(1)}(0) \\
\boldsymbol{D}_{i} & =\left[\begin{array}{ll}
\boldsymbol{M}_{12}^{(i)}\left(-h_{i}\right) & -\boldsymbol{M}_{11}^{(i+1)}\left(-h_{i}\right) \\
\boldsymbol{M}_{22}^{(i)}\left(-h_{i}\right) & -\boldsymbol{M}_{21}^{(i+1)}\left(-h_{i}\right)
\end{array}\right], \quad i=1,2, \ldots, n-1, \\
\boldsymbol{D}_{n} & =\boldsymbol{M}_{22}^{(n)}\left(-h_{n}\right)
\end{array}\right\}
$$


the non-zero block elements of upper triangular matrix $\boldsymbol{U}$ are

$$
\begin{aligned}
\boldsymbol{U}_{0} & =\left(\begin{array}{ll}
-\boldsymbol{M}_{22}^{(n)}(0) & \mathbf{0}_{2 \times 2}
\end{array}\right), \\
\boldsymbol{U}_{i} & =\left(\begin{array}{ll}
-\boldsymbol{M}_{12}^{(i+1)}\left(-h_{i}\right) & \mathbf{0}_{2 \times 2} \\
-\boldsymbol{M}_{22}^{(i+1)}\left(-h_{i}\right) & \mathbf{0}_{2 \times 2}
\end{array}\right), \quad i=1,2, \ldots, n-2, \\
\boldsymbol{U}_{n-1} & =\left(\begin{array}{l}
-\boldsymbol{M}_{12}^{(n)}\left(-h_{n-1}\right) \\
-\boldsymbol{M}_{22}^{(n)}\left(-h_{n-1}\right)
\end{array}\right)
\end{aligned}
$$

and the non-zero blocks for lower triangular matrix $\boldsymbol{L}$ are

$$
\begin{aligned}
\boldsymbol{L}_{1} & =\left(\begin{array}{l}
\boldsymbol{M}_{11}^{(1)}\left(-h_{1}\right) \\
\boldsymbol{M}_{21}^{(1)}\left(-h_{1}\right)
\end{array}\right), \\
\boldsymbol{L}_{i} & =\left(\begin{array}{ll}
\mathbf{0}_{2 \times 2} & \boldsymbol{M}_{11}^{(i)}\left(-h_{i}\right) \\
\mathbf{0}_{2 \times 2} & \boldsymbol{M}_{21}^{(i)}\left(-h_{i}\right)
\end{array}\right), \quad i=2,3, \ldots, n-1, \\
\boldsymbol{L}_{n} & =\left(\begin{array}{ll}
\mathbf{0}_{2 \times 2} & \boldsymbol{M}_{21}^{(n)}\left(-h_{n}\right)
\end{array}\right) .
\end{aligned}
$$

Note that the diagonal block matrix $\boldsymbol{D}$ is a non-singular matrix. The stacked matrix equation can be solved directly by

$$
c=M^{-1} \hat{\boldsymbol{t}} .
$$

Once the global field vector $\boldsymbol{c}$ is obtained, the response functions in each layer can be determined immediately. By arranging the response functions in each layer into a response vector, this vector can be related to the globe field vector with a phase-related receiver matrix $\boldsymbol{R}_{c v}$. For example, if the response functions for $u$, $v, \sigma_{y y}$ and $\sigma_{x y}$ in each layer are concerned, the response vector should be defined as

$$
\hat{\boldsymbol{b}}(y ; \xi, p)=\left(\left(\begin{array}{c}
\hat{u}^{(1)}(y) \\
\hat{v}^{(1)}(y) \\
\hat{\sigma}_{y y}^{(1)}(y) \\
\hat{\sigma}_{x y}^{(1)}(y)
\end{array}\right)^{\mathrm{T}} \quad\left(\begin{array}{c}
\hat{u}^{(2)}(y) \\
\hat{v}^{(2)}(y) \\
\hat{\sigma}_{y y}^{(2)}(y) \\
\hat{\sigma}_{x y}^{(2)}(y)
\end{array}\right)^{\mathrm{T}} \quad \cdots \quad\left(\begin{array}{c}
\hat{u}^{(n-1)}(y) \\
\hat{v}^{(n-1)}(y) \\
\hat{\sigma}_{y y}^{(n-1)}(y) \\
\hat{\sigma}_{x y}^{(n-1)}(y)
\end{array}\right)^{\mathrm{T}} \quad\left(\begin{array}{c}
\hat{u}^{(n)}(y) \\
\hat{v}^{(n)}(y) \\
\hat{\sigma}_{y y}^{(n)}(y) \\
\hat{\sigma}_{x y}^{(n)}(y)
\end{array}\right)^{\mathrm{T}}\right)^{\mathrm{T}} .
$$

Thus the global field vector is related to the response vector by

$$
\hat{\boldsymbol{b}}(y ; \xi, p)=\boldsymbol{R}_{\boldsymbol{c v}}(y) \boldsymbol{M}^{-1} \hat{\boldsymbol{t}},
$$


where the global phase-related receiver matrix is given by (in view of equation (3.8))

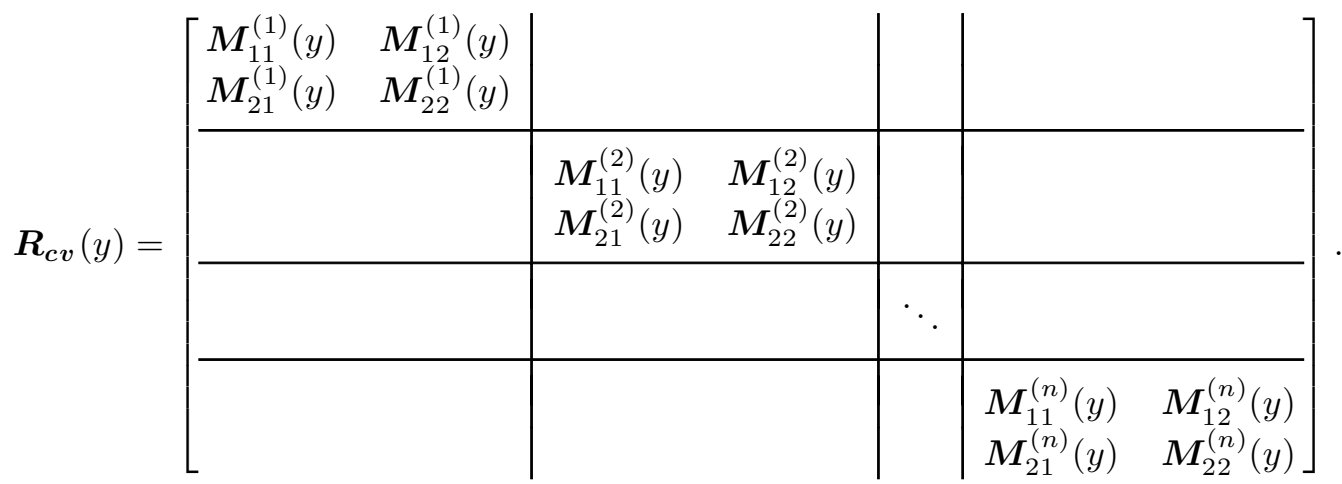

Note that the elements of the phase-related receiver matrix $\boldsymbol{R}_{c v}$ are independent of the loading conditions $\hat{\boldsymbol{t}}$. Furthermore, they change correspondingly if the response function vector changes. The flexibility in choosing $\boldsymbol{R}_{\boldsymbol{c} v}$ and $\hat{\boldsymbol{b}}$ facilitates the solution for a multiple receiver system.

With the transformed solution at hand, the inverse transform should be performed to obtain the transient solution in time domain. The inverse transform method was discussed in some detail in the book written by Ewing et al. (1957). The inversion of Laplace transform involves a summation of residues which are infinite in number. Hence the accuracy of the final value depends on the number of terms taken in the series.

As an alternative way to solve the problem, the theory of generalized ray was designated to calculate the early response of the multi-layered medium by a different approach obviating the solving of the boundary-value problem. The relations between this method and the boundary-value problem, however, are obscure. In the method of generalized ray, the transient response of the layered medium is decomposed into an infinite number of rays which arrive at the receiver along a generalized ray path in sequence. Solutions based on generalized ray theory is completed with the application of Cagniard's method. Since the transient response for the layered medium is exact up to the arrival time of the next ray, only a finite number of rays are involved in the early-time solution. For the long-time response at a receiver in the layered medium, however, difficulty arises in determining the large number of generalized ray paths along which the rays arrived at the receiver. Therefore, an effective method will be provided here to obtain the ray solution directly from the boundary-value problem for the layered medium.

The coefficient matrix $\boldsymbol{M}$ is rewritten in an alternative form by extracting the diagonal block matrix $\boldsymbol{D}$ out of the expression as

$$
\boldsymbol{M}=\boldsymbol{D}(\boldsymbol{I}-\boldsymbol{R}),
$$

where $\boldsymbol{R}$ is given by

$$
\boldsymbol{R}=-\boldsymbol{D}^{-1}(\boldsymbol{L}+\boldsymbol{U}),
$$


or, alternatively,

$$
\boldsymbol{R}=\left[\begin{array}{cccc}
\mathbf{0}_{2 \times 2} & -\boldsymbol{D}_{0}^{-1} \boldsymbol{U}_{0} & & \\
-\boldsymbol{D}_{1}^{-1} \boldsymbol{L}_{1} & \mathbf{0}_{4 \times 4} & -\boldsymbol{D}_{1}^{-1} \boldsymbol{U}_{1} & \\
& -\boldsymbol{D}_{2}^{-1} \boldsymbol{L}_{2} & \mathbf{0}_{4 \times 4} & \ddots \\
& & \ddots & \ddots \\
& & & \ddots
\end{array}\right.
$$

$$
\left.\begin{array}{ccc} 
& & \\
\ddots & & \\
\mathbf{0}_{4 \times 4} & -\boldsymbol{D}_{n-2}^{-1} \boldsymbol{U}_{n-2} & \\
-\boldsymbol{D}_{n-1}^{-1} \boldsymbol{L}_{n-1} & \mathbf{0}_{4 \times 4} & -\boldsymbol{D}_{n-1}^{-1} \boldsymbol{U}_{n-1} \\
& -\boldsymbol{D}_{n}^{-1} \boldsymbol{L}_{n} & \mathbf{0}_{2 \times 2}
\end{array}\right] .
$$

From (4.9) and (4.13), the global field vector $\boldsymbol{c}$ is then obtained by

$$
\boldsymbol{c}=(\boldsymbol{I}-\boldsymbol{R})^{-1} \boldsymbol{s},
$$

where the source vector $s$ is given by

$$
s=D^{-1} \hat{\boldsymbol{t}},
$$

or, in a component form,

$$
\boldsymbol{s}=\left(\begin{array}{c}
\boldsymbol{D}_{0}^{-1} \hat{\boldsymbol{t}}^{[0]} \\
\boldsymbol{D}_{1}^{-1} \hat{\boldsymbol{t}}^{[1]} \\
\vdots \\
\boldsymbol{D}_{n-1}^{-1} \hat{\boldsymbol{t}}^{[n-1]} \\
\boldsymbol{D}_{n}^{-1} \hat{\boldsymbol{t}}^{[n]}
\end{array}\right) .
$$

To examine the localized source effect, a typical loading $\boldsymbol{t}^{[i]}$ is applied at the interface $y=-h_{i}$ separating the $i$ th and $(i+1)$ th layers. Source waves generated directly by the applied loading are propagating in these two layers as if they are propagating in two joint half-spaces as shown in figure 2. Since only the outgoing waves from the interface are generated, the field coefficient vectors

$$
\boldsymbol{c}_{-}^{(i)} \text { and } \boldsymbol{c}_{+}^{(i+1)}
$$

for incoming waves will vanish. The interface condition in the transformed domain yields

$$
\left(\begin{array}{ll}
\boldsymbol{M}_{12}^{(i)}\left(-h_{i}\right) & -\boldsymbol{M}_{11}^{(i+1)}\left(-h_{i}\right) \\
\boldsymbol{M}_{22}^{(i)}\left(-h_{i}\right) & -\boldsymbol{M}_{21}^{(i+1)}\left(-h_{i}\right)
\end{array}\right)\left(\begin{array}{c}
c_{+}^{(i)} \\
\boldsymbol{c}_{-}^{(i+1)}
\end{array}\right)=\hat{\boldsymbol{t}}^{[i]} .
$$






Figure 2. Waves generated by an interfacial loading.

Therefore, the non-zero field coefficient vectors $\boldsymbol{c}_{+}^{(i)}$ and $\boldsymbol{c}_{-}^{(i+1)}$ are obtained as

$$
\left(\begin{array}{c}
c_{+}^{(i)} \\
\boldsymbol{c}_{-}^{(i+1)}
\end{array}\right)=\boldsymbol{D}_{i}^{-1} \hat{\boldsymbol{t}}^{[i]},
$$

where the coefficient matrices $\boldsymbol{D}_{i}$ are exactly the same as that given in (4.6). The physical meaning of the source vector $s$ in (4.17) now becomes clear. Each element of the vector represents the source waves generated by the applied loadings. In comparison with the source function designated for the theory of generalized ray, the source vector $s$ is phase related.

By the expansion of the inversion matrix of $(\boldsymbol{I}-\boldsymbol{R})$ in (4.16) into a power matrix series of $\boldsymbol{R}$, we obtain

$$
\boldsymbol{c}=\sum_{i=0}^{\infty} \boldsymbol{R}^{i} \boldsymbol{s}
$$

The response vector $\hat{\boldsymbol{b}}$ in (4.11) can be expressed as

$$
\hat{\boldsymbol{b}}(y ; \xi, p)=\boldsymbol{R}_{\boldsymbol{c} \boldsymbol{v}}(y) \sum_{i=0}^{\infty} \boldsymbol{R}^{i} \boldsymbol{s} .
$$

The convergence of the series will be ensured in the following section by considering the physical meaning of $\boldsymbol{R}$.

\section{The phase-related reflection and transmission matrix}

Following the basic idea of the method of generalized ray which was proposed by Spencer (1960), the boundary-value problem for a single interface between two halfspaces (as shown in figure 3) is considered to construct the reflected and transmitted coefficients of the two media adjacent to the interface. The waves which are propagating toward the interface $(i)$ from the upper medium $(i)$ will be considered first and are shown in figure 3a. The downgoing incident field vector in the medium $(i)$ is denoted as $\boldsymbol{c}_{0-}^{(i)}$. When the downgoing waves arrive at the interface, the upgoing reflected waves in the medium $(i)$ and the downgoing transmitted waves in the medium $(i+1)$ will be generated. The reflected and transmitted field vectors are denoted as

$$
\boldsymbol{c}_{1+}^{(i)} \text { and } \boldsymbol{c}_{1-}^{(i+1)}
$$


(a)

$$
c_{0-}^{(i)} \downarrow c_{1+}^{(i)}
$$

interface $(i)$ $y=-h_{i}$

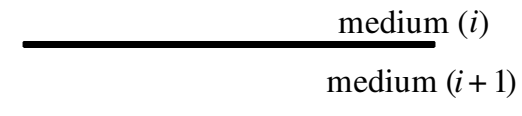

$\boldsymbol{c}_{1-}^{(i+1)}$

(b)

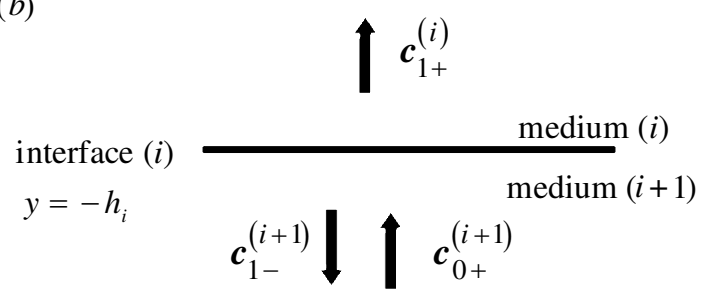

Figure 3. Reflection and transmission of waves by a single interface. (a) Incident wave in the direction of decreasing $y$. (b) Incident wave in the direction of increasing $y$.

respectively. Note that additional subscripts 0 and 1 for the field vectors indicate the incident waves and the reflected or transmitted waves, respectively.

The continuity condition at interface $(i)$ yields

$$
\left(\begin{array}{ll}
\boldsymbol{M}_{11}^{(i)}\left(-h_{i}\right) & \boldsymbol{M}_{12}^{(i)}\left(-h_{i}\right) \\
\boldsymbol{M}_{21}^{(i)}\left(-h_{i}\right) & \boldsymbol{M}_{22}^{(i)}\left(-h_{i}\right)
\end{array}\right)\left(\begin{array}{l}
\boldsymbol{c}_{0-}^{(i)} \\
\boldsymbol{c}_{1+}^{(i)}
\end{array}\right)=\left(\begin{array}{l}
\boldsymbol{M}_{11}^{(i+1)}\left(-h_{i}\right) \\
\boldsymbol{M}_{21}^{(i+1)}\left(-h_{i}\right)
\end{array}\right)\left(\boldsymbol{c}_{1-}^{(i+1)}\right) .
$$

Solving for $(5.1)$, the reflected and transmitted field vectors are expressed in terms of the incident field vector as follows,

$$
\begin{aligned}
\left(\begin{array}{c}
\boldsymbol{c}_{1+}^{(i)} \\
\boldsymbol{c}_{1-}^{(i+1)}
\end{array}\right) & =-\boldsymbol{D}_{i}^{-1} \boldsymbol{L}_{i}\left(\begin{array}{c}
\mathbf{0} \\
\boldsymbol{c}_{0-}^{(i)}
\end{array}\right) \\
& =\left(\begin{array}{cc}
\mathbf{0} & \boldsymbol{R}_{i / i+1} \\
\mathbf{0} & \boldsymbol{T}_{i / i+1}
\end{array}\right)\left(\begin{array}{c}
\mathbf{0} \\
\boldsymbol{c}_{0-}^{(i)}
\end{array}\right),
\end{aligned}
$$

where the matrices $\boldsymbol{D}_{i}$ and $\boldsymbol{L}_{i}$ are the same as those given in (4.6) and (4.8), respectively. The term $-\boldsymbol{D}_{i}^{-1} \boldsymbol{L}_{i}$ is exactly the same as the element at the $i$ th level of the matrix $\boldsymbol{R}$ as given in (4.15). The phase-related reflection matrix $\boldsymbol{R}_{i / i+1}$ at the interface $(i)\left(y=-h_{i}\right)$ is expressed as

$$
\boldsymbol{R}_{i / i+1}=\left[\begin{array}{cc}
R_{p p} \mathrm{e}^{-2 p \gamma_{\mathrm{L}}^{(i)} h_{i}} & R_{s p} \mathrm{e}^{-p\left(\gamma_{\mathrm{L}}^{(i)}+\gamma_{\mathrm{T}}^{(i)}\right) h_{i}} \\
R_{p s} \mathrm{e}^{-p\left(\gamma_{\mathrm{L}}^{(i)}+\gamma_{\mathrm{T}}^{(i)}\right) h_{i}} & R_{s s} \mathrm{e}^{-2 p \gamma_{\mathrm{T}}^{(i)} h_{i}}
\end{array}\right]
$$

the phase-related transmission matrix $\boldsymbol{T}_{i / i+1}$ is

$$
\boldsymbol{T}_{i / i+1}=\left[\begin{array}{ll}
T_{p p} \mathrm{e}^{-p\left(\gamma_{\mathrm{L}}^{(i)}-\gamma_{\mathrm{L}}^{(i+1)}\right) h_{i}} & T_{s p} \mathrm{e}^{-p\left(\gamma_{\mathrm{T}}^{(i)}-\gamma_{\mathrm{L}}^{(i+1)}\right) h_{i}} \\
T_{p s} \mathrm{e}^{-p\left(\gamma_{\mathrm{L}}^{(i)}-\gamma_{\mathrm{T}}^{(i+1)}\right) h_{i}} & T_{s s} \mathrm{e}^{-p\left(\gamma_{\mathrm{T}}^{(i)}-\gamma_{\mathrm{T}}^{(i+1)}\right) h_{i}}
\end{array}\right] .
$$


The functions $R_{p p}, R_{p s}, R_{s p}$ and $R_{s s}$ are referred to the generalized reflection coefficients. Likewise, $T_{p p}, T_{p s}, T_{s p}$ and $T_{s s}$ are generalized transmission coefficients. They are expressed as

$$
\left.\begin{array}{llll}
R_{p p}=\frac{r_{p p}}{\Delta}, & R_{s p}=\frac{r_{s p}}{\Delta}, & R_{p s}=\frac{r_{p s}}{\Delta}, & R_{s s}=\frac{r_{s s}}{\Delta}, \\
T_{p p}=\frac{t_{p p}}{\Delta}, & T_{s p}=\frac{t_{s p}}{\Delta}, & T_{p s}=\frac{t_{p s}}{\Delta}, & T_{s s}=\frac{t_{s s}}{\Delta},
\end{array}\right\}
$$

where

$$
\begin{aligned}
\Delta=- & \left(\left(A_{(i+1)}^{2}+4 \xi^{2} \gamma_{\mathrm{L}(i+1)} \gamma_{\mathrm{T}(i+1)}\right)\left(\xi^{2}+\gamma_{\mathrm{L}(i)} \gamma_{\mathrm{T}(i)}\right) \bar{\mu}^{2}\right. \\
& +\left(s_{\mathrm{T}(i)}^{2} s_{\mathrm{T}(i+1)}^{2}\left(\gamma_{\mathrm{L}(i)} \gamma_{\mathrm{T}(i+1)}+\gamma_{\mathrm{L}(i+1)} \gamma_{\mathrm{T}(i)}\right)\right. \\
& \left.-2 \xi^{2}\left(A_{(i)}-2 \gamma_{\mathrm{L}(i)} \gamma_{\mathrm{T}(i)}\right)\left(A_{(i+1)}-2 \gamma_{\mathrm{L}(i+1)} \gamma_{\mathrm{T}(i+1)}\right)\right) \bar{\mu} \\
& \left.+\left(A_{(i)}^{2}+4 \xi^{2} \gamma_{\mathrm{L}(i)} \gamma_{\mathrm{T}(i)}\right)\left(\xi^{2}+\gamma_{\mathrm{L}(i+1)} \gamma_{\mathrm{T}(i+1)}\right)\right)
\end{aligned}
$$

in which

$$
\bar{\mu}=\frac{\mu_{(i+1)}}{\mu_{(i)}}, \quad A_{(i)}=s_{\mathrm{T}(i)}^{2}-2 \xi^{2} .
$$

In (5.5), the numerators in the expression of the generalized reflection coefficients are given by

$$
\begin{aligned}
r_{p p}=- & \left(A_{(i+1)}^{2}+4 \xi^{2} \gamma_{\mathrm{L}(i+1)} \gamma_{\mathrm{T}(i+1)}\right)\left(\xi^{2}-\gamma_{\mathrm{L}(i)} \gamma_{\mathrm{T}(i)}\right) \bar{\mu}^{2} \\
& -\left(s_{\mathrm{T}(i)}^{2} s_{\mathrm{T}(i+1)}^{2}\left(\gamma_{\mathrm{L}(i+1)} \gamma_{\mathrm{T}(i)}-\gamma_{\mathrm{L}(i)} \gamma_{\mathrm{T}(i+1)}\right)\right. \\
& \left.-2 \xi^{2}\left(A_{(i)}+2 \gamma_{\mathrm{L}(i)} \gamma_{\mathrm{T}(i)}\right)\left(A_{(i+1)}-2 \gamma_{\mathrm{L}(i+1)} \gamma_{\mathrm{T}(i+1)}\right)\right) \bar{\mu} \\
& -\left(A_{(i)}^{2}-4 \xi^{2} \gamma_{\mathrm{L}(i)} \gamma_{\mathrm{T}(i)}\right)\left(\xi^{2}+\gamma_{\mathrm{L}(i+1)} \gamma_{\mathrm{T}(i+1)}\right), \\
r_{p s}=2 \xi \gamma_{\mathrm{L}(i)}\left[\left(A_{(i+1)}^{2}+4 \xi^{2} \gamma_{\mathrm{L}(i+1)} \gamma_{\mathrm{T}(i+1)}\right) \bar{\mu}^{2}\right. & \\
& \left.-\left(A_{(i)}-2 \xi^{2}\right)\left(A_{(i+1)}-2 \gamma_{\mathrm{L}(i+1)} \gamma_{\mathrm{T}(i+1)}\right) \bar{\mu}-2 A_{(i)}\left(\xi^{2}+\gamma_{\mathrm{L}(i+1)} \gamma_{\mathrm{T}(i+1)}\right)\right], \\
r_{s p}=- & 2 \xi \gamma_{\mathrm{T}(i)}\left[\left(A_{(i+1)}^{2}+4 \xi^{2} \gamma_{\mathrm{L}(i+1)} \gamma_{\mathrm{T}(i+1)}\right) \bar{\mu}^{2}\right. \\
& \left.-\left(A_{(i)}-2 \xi^{2}\right)\left(A_{(i+1)}-2 \gamma_{\mathrm{L}(i+1)} \gamma_{\mathrm{T}(i+1)}\right) \bar{\mu}-2 A_{(i)}\left(\xi^{2}+\gamma_{\mathrm{L}(i+1)} \gamma_{\mathrm{T}(i+1)}\right)\right], \\
r_{s s}=- & \left(A_{(i+1)}^{2}+4 \xi^{2} \gamma_{\mathrm{L}(i+1)} \gamma_{\mathrm{T}(i+1)}\right)\left(\xi^{2}-\gamma_{\mathrm{L}(i)} \gamma_{\mathrm{T}(i)}\right) \bar{\mu}^{2} \\
& -\left(s_{\mathrm{T}(i)}^{2} s_{\mathrm{T}(i+1)}^{2}\left(\gamma_{\mathrm{L}(i)} \gamma_{\mathrm{T}(i+1)}-\gamma_{\mathrm{L}(i+1)} \gamma_{\mathrm{T}(i)}\right)\right. \\
& \left.-2 \xi^{2}\left(A_{(i)}+2 \gamma_{\mathrm{L}(i)} \gamma_{\mathrm{T}(i)}\right)\left(A_{(i+1)}-2 \gamma_{\mathrm{L}(i+1)} \gamma_{\mathrm{T}(i+1)}\right)\right) \bar{\mu} \\
& -\left(A_{(i)}^{2}-4 \xi^{2} \gamma_{\mathrm{L}(i)} \gamma_{\mathrm{T}(i)}\right)\left(\xi^{2}+\gamma_{\mathrm{L}(i+1)} \gamma_{\mathrm{T}(i+1)}\right),
\end{aligned}
$$

and the numerators in the expression of generalized transmission coefficients are

$$
\begin{aligned}
t_{p p} & =2 s_{\mathrm{T}(i)}^{2} \gamma_{\mathrm{L}(i)}\left(\left(A_{(i+1)} \gamma_{\mathrm{T}(i)}+2 \xi^{2} \gamma_{\mathrm{T}(i+1)}\right) \bar{\mu}+\left(A_{(i)} \gamma_{\mathrm{T}(i+1)}+2 \xi^{2} \gamma_{\mathrm{T}(i)}\right)\right), \\
t_{p s} & =2 \xi s_{\mathrm{T}(i)}^{2} \gamma_{\mathrm{L}(i)}\left(\left(A_{(i+1)}-2 \gamma_{\mathrm{L}(i+1)} \gamma_{\mathrm{T}(i)}\right) \bar{\mu}-\left(A_{(i)}-2 \gamma_{\mathrm{L}(i+1)} \gamma_{\mathrm{T}(i)}\right)\right), \\
t_{s p} & =-2 \xi s_{\mathrm{T}(i)}^{2} \gamma_{\mathrm{T}(i)}\left(\left(A_{(i+1)}-2 \gamma_{\mathrm{L}(i)} \gamma_{\mathrm{T}(i+1)}\right) \bar{\mu}-\left(A_{(i)}-2 \gamma_{\mathrm{L}(i)} \gamma_{\mathrm{T}(i+1)}\right)\right), \\
t_{s s} & =2 s_{\mathrm{T}(i)}^{2} \gamma_{\mathrm{T}(i)}\left(\left(A_{(i+1)} \gamma_{\mathrm{L}(i)}+2 \xi^{2} \gamma_{\mathrm{L}(i+1)}\right) \bar{\mu}+\left(A_{(i)} \gamma_{\mathrm{L}(i+1)}+2 \xi^{2} \gamma_{\mathrm{L}(i)}\right)\right)
\end{aligned}
$$


By setting $\Delta$ equal to zero in (5.6), i.e.

$$
\Delta=0,
$$

we have the characteristic equation for Stoneley waves at the interface of two dissimilar isotropic solids. If the medium $(i+1)$ is a vacuum, the transmission coefficients are zero and the reflection coefficients at a free surface will be obtained by setting $\bar{\mu}=0$ in (5.5). The characteristic equation for the Stoneley wave in (5.9) reduces to that for the Rayleigh wave. As another limiting case, if medium $(i+1)$ is a rigid body, the displacement of medium $(i+1)$ is zero at the interface. The reflection coefficients are obtained by taking the limit $\bar{\mu} \rightarrow \infty$ in (5.5), while the transmission coefficients are absent.

In view of (5.2), the phase-related reflected matrix $\boldsymbol{R}_{i / i+1}$ characterizes the transfer relations between the incident and the reflected wave fields. If there is an incident pressure wave (in the $i$ th layer) propagating toward the interface between the $i$ th and $(i+1)$ th layer, the coefficient of reflected pressure wave can be obtained immediately by multiplying the coefficient of incident pressure wave with $\left[\boldsymbol{R}_{i / i+1}\right]_{11}$. The coefficient of the reflected shear wave can be obtained from the incident coefficient by multiplying $\left[\boldsymbol{R}_{i / i+1}\right]_{12}$. Similarly, if the incident wave is a shear wave, the coefficients of reflected pressure and shear waves can be constructed by multiplying the coefficient of incident shear wave with $\left[\boldsymbol{R}_{i / i+1}\right]_{21}$ and $\left[\boldsymbol{R}_{i / i+1}\right]_{22}$, respectively.

The phase-related transmission matrix $\boldsymbol{T}_{i / i+1}$ dominates the transfer relations between the incident and the transmitted wave fields. The wave potentials for the transmission waves can be obtained easily by multiplying the coefficients of incident wave potentials with correspondent elements in $\boldsymbol{T}_{i / i+1}$. The physical interpretation will be discussed in detail in the second part of this paper through an example of a two-layered medium.

Suppose there is an incident wave, represented by a field vector $\boldsymbol{c}_{0+}^{(i+1)}$, which travels in the direction of increasing $y$ as shown in figure $3 b$. In other words, there is a source wave $\boldsymbol{c}_{0+}^{(i+1)}$ propagating upward toward the interface $(i)$. By the application of the continuity conditions at the interface, the reflected field vector $\boldsymbol{c}_{1-}^{(i+1)}$ and the transmitted one $\boldsymbol{c}_{1+}^{(i)}$ are expressed in terms of incident field vector as follows

$$
\left.\begin{array}{rl}
\left(\begin{array}{c}
\boldsymbol{c}_{1+}^{(i)} \\
\boldsymbol{c}_{1-}^{(i+1)}
\end{array}\right) & =-\boldsymbol{D}_{i}^{-1} \boldsymbol{U}_{i}\left(\begin{array}{c}
\boldsymbol{c}_{0+}^{(i+1)} \\
\mathbf{0}
\end{array}\right) \\
& =\left(\begin{array}{ll}
\boldsymbol{T}_{i+1 / i} & \mathbf{0} \\
\boldsymbol{R}_{i+1 / i} & \mathbf{0}
\end{array}\right)\left(\begin{array}{c}
\boldsymbol{c}_{0+}^{(i+1)} \\
\mathbf{0}
\end{array}\right),
\end{array}\right\}
$$

where $\boldsymbol{D}_{i}$ and $\boldsymbol{U}_{i}$ are given in (4.6) and (4.7), respectively. The phase-related reflected matrix $\boldsymbol{R}_{i+1 / i}$ becomes

$$
\boldsymbol{R}_{i+1 / i}=\left[\begin{array}{cc}
R^{p p} \mathrm{e}^{-2 p \gamma_{\mathrm{L}}^{(i+1)} h_{i}} & R^{s p} \mathrm{e}^{-p\left(\gamma_{\mathrm{L}}^{(i+1)}+\gamma_{\mathrm{T}}^{(i+1)}\right) h_{i}} \\
R^{p s} \mathrm{e}^{-p\left(\gamma_{\mathrm{L}}^{(i+1)}+\gamma_{\mathrm{T}}^{(i+1)}\right) h_{i}} & R^{s s} \mathrm{e}^{-2 p \gamma_{\mathrm{T}}^{(i+1)} h_{i}}
\end{array}\right] ;
$$

the phase-related transmission matrix $\boldsymbol{T}_{i+1 / i}$ is

$$
\boldsymbol{T}_{i+1 / i}=\left[\begin{array}{ll}
T^{p p} \mathrm{e}^{p\left(\gamma_{\mathrm{L}}^{(i)}-\gamma_{\mathrm{L}}^{(i+1)}\right) h_{i}} & T^{s p} \mathrm{e}^{p\left(\gamma_{\mathrm{T}}^{(i)}-\gamma_{\mathrm{L}}^{(i+1)}\right) h_{i}} \\
T^{p s} \mathrm{e}^{p\left(\gamma_{\mathrm{L}}^{(i)}-\gamma_{\mathrm{T}}^{(i+1)}\right) h_{i}} & T^{s s} \mathrm{e}^{p\left(\gamma_{\mathrm{T}}^{(i)}-\gamma_{\mathrm{T}}^{(i+1)}\right) h_{i}}
\end{array}\right] .
$$


For the incident wave propagating in the direction of increasing $y$, the reflection and transmission coefficients are given by

$$
\left.\begin{array}{llll}
R_{p p}=R^{p p}, & R_{s s}=R^{s s}, & T_{p p}=T^{p p}, & T_{s s}=T^{s s}, \\
R_{p s}=-R^{p s}, & R_{s p}=-R^{s p}, & T_{p s}=-T^{p s}, & T_{s p}=-T^{s p} .
\end{array}\right\}
$$

The phase-related reflection and transmission coefficient matrices $\boldsymbol{R}_{i+1 / i}$ and $\boldsymbol{T}_{i+1 / i}$ characterize the solution of reflected and transmitted waves which are generated by the interface $(i)$ for incident waves that are propagating in the medium $(i+1)$.

By stacking all the localized phase-related reflection and transmission matrices for each single interface, a globe reflection and transmission matrix can be constructed to characterize the waves propagating in the multi-layered medium. The field vector $c_{1}$ generated by the incident field vector $\boldsymbol{s}$ (the source waves) is thus given by

$$
c_{1}=R s,
$$

where the global phase-related reflection and transmission matrix $\boldsymbol{R}$ is exactly the same form as that given in (4.15), and can be rewritten in terms of the local reflection and transmission matrices as follows:

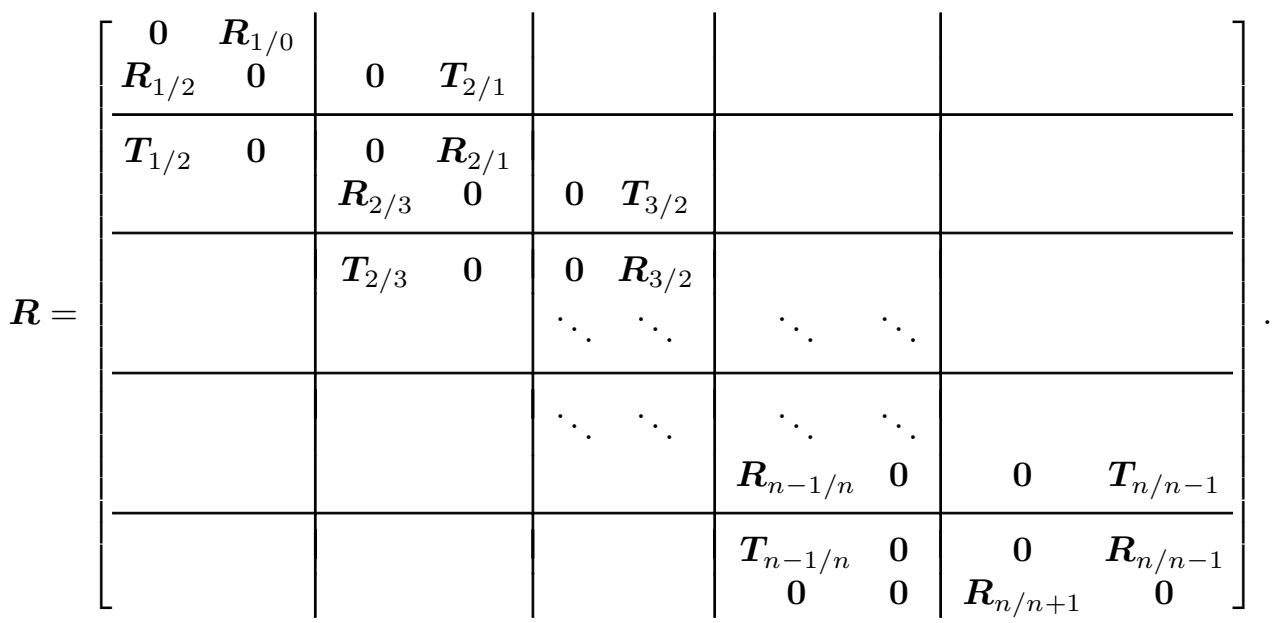

Note that the field vector $\boldsymbol{c}_{1}$ represents all the scatter waves induced by the source waves that are reflected by, or transmitted through, the interface once. The scatter waves represented by $\boldsymbol{c}_{1}$ become the succeeding incident waves for the medium, and the secondary scatter waves (which are represented by $\boldsymbol{c}_{2}=\boldsymbol{R} \boldsymbol{c}_{1}$ ) will be generated and considered as the incident waves for the next group of waves. The complete wave field in the layered medium are thus decomposed into an infinite number of wave groups which are generated in successive order.

A synthesis of all wave groups generated by source waves in sequence leads to the total wave field in the layered medium, i.e.

$$
\boldsymbol{c}=\sum_{i=0}^{\infty} \boldsymbol{R}^{i} \boldsymbol{s} .
$$

This result is exactly the same as the one given in (4.20). The term $\boldsymbol{R}^{i} \boldsymbol{s}$ represents a group of waves which is either reflected by, or transmitted through, the interface $i$ 
times. Since the sum of reflection and transmission coefficients in each row of matrix $\boldsymbol{R}$ is less than or equal to unity, all the eigenvalues of matrix $\boldsymbol{R}$ are allocated in a unit circle in the complex plane. The convergence of the matrix series is ensured. However, this expansion is applicable to practical problems, since only first few terms out of infinite sum are relevant for any given time of interest.

In the layered-medium problem, the solution for interface loading conditions is now decomposed into infinite groups of waves, each represented by a term in the infinite matrix series in (4.20). The phase-related reflection and transmission matrix $\boldsymbol{R}$ characterizes the multiple reflections of all waves within the medium, and the source vector $s$ specifies the source waves generated by the applied loadings at the interface and lateral surfaces. For the response quantities of the layered medium, such as displacement and stress components at a receiver, equation (4.21) gives the solution by multiplying the solution of displacement potential in the transformed domain with the phase-related receiver matrix $\boldsymbol{R}_{\boldsymbol{c} v}$ that is adjustable and is determined by the response function we are interested.

The source vector $\boldsymbol{s}$, the reflection and transmission matrix $\boldsymbol{R}$ and the receiver matrix $\boldsymbol{R}_{\boldsymbol{c} v}$ are independent of each other. When the loading conditions are changed, the expressions of $\boldsymbol{R}$ and $\boldsymbol{R}_{\boldsymbol{c} \boldsymbol{v}}$ will still be the same. The independence of $\boldsymbol{R}_{\boldsymbol{c} \boldsymbol{v}}, \boldsymbol{R}$ and $\boldsymbol{s}$ implies that only the source vector $\boldsymbol{s}$ should be constructed in solving different problems. In the next section, the proposed method will be extended to solve the problem with a loading that is applied in the interior of the layered medium.

\section{The interior source problem}

In this section, the transient response of the layered medium subjected to dynamic loadings which are located within layers instead of at the interface will be considered. The solution can be obtained by introducing artificial interfaces at the applied-loading locations. In this way, the size of matrix $\boldsymbol{R}$ will be increased. As an alternative, the solution can be constructed easily by modifying the source vector $s$ presented in (4.20) in considering the physical meaning of the reflection and transmission matrix $\boldsymbol{R}$ in (5.15).

Since the representation of the source waves in the infinite domain depends on the location of receiver, the source term in the matrix solution should be separated from the summation and will be denoted as a vector $\boldsymbol{s}_{0}^{*}$ in the following expression. The source emits waves propagate in two directions, which will become the incident waves in the successive reflection and transmission by the interfaces. The source vector $s$ in (5.14) includes the waves in both directions and is denoted by $\boldsymbol{s}^{*}$ in order to distinguish between the source function of interface loading and body loading. The succeeding reflected or transmission waves are thus obtained by multiplying the matrix $\boldsymbol{R}$ with $\boldsymbol{s}^{*}$. For example, the transformed solution for a body source at level $y=-h_{s_{i}}$ in the $i$ th layer is expressed as follows

$$
\hat{\boldsymbol{b}}(y ; \xi, p)=\boldsymbol{R}_{\boldsymbol{c} \boldsymbol{v}}(y) \boldsymbol{s}_{0}^{*}+\boldsymbol{R}_{\boldsymbol{c v}}(y) \sum_{i=1}^{\infty} \boldsymbol{R}^{i} \boldsymbol{s}^{*},
$$

where $\boldsymbol{s}_{0}^{*}$ is defined as

$$
\begin{array}{ll}
\boldsymbol{s}_{0}^{*}=\left(0,0, \ldots, 0, \boldsymbol{s}_{+}^{(i)}\left(-h_{s_{i}}\right), \ldots, 0\right)^{\mathrm{T}} \quad \text { for }-h_{i}>y>-h_{s_{i}}>h_{i}, \\
\boldsymbol{s}_{0}^{*}=\left(0,0, \ldots, \boldsymbol{s}_{-}^{(i)}\left(-h_{s_{i}}\right), 0, \ldots, 0,0\right)^{\mathrm{T}} \quad \text { for }-h_{i}>-h_{s_{i}}>y>h_{i}
\end{array}
$$


Table 1. The source functions ${ }^{\mathrm{a}}$ in infinite domain at $y=-h_{s}$

\begin{tabular}{|c|c|c|c|c|c|c|}
\hline types of loading & & $F(p)^{\mathrm{b}}$ & $\phi_{0-}(\xi)^{\mathrm{a}}$ & $\psi_{0-}(\xi)^{\mathrm{a}}$ & $\phi_{0+}(\xi)^{\mathrm{a}}$ & $\psi_{0+}(\xi)^{\mathrm{a}}$ \\
\hline \multicolumn{7}{|c|}{ concentrated forces } \\
\hline vertical & $\uparrow$ & $\frac{\sigma_{0}}{2 \mu n^{2} s^{2}}$ & 1 & $\frac{\xi}{\gamma_{T}}$ & -1 & $\frac{\xi}{\gamma_{T}}$ \\
\hline horizontal & $\rightarrow$ & $\frac{\sigma_{0}}{2 \mu p^{2} s_{\mathrm{T}}^{2}}$ & $-\frac{\xi}{\gamma_{\mathrm{L}}}$ & 1 & $-\frac{\xi}{\gamma_{\mathrm{L}}}$ & -1 \\
\hline \multicolumn{7}{|c|}{ concentrated double forces } \\
\hline vertical & $\uparrow$ & $\frac{d_{0}}{2 \mu p s_{\mathrm{T}}^{2}}$ & $\gamma_{\mathrm{L}}$ & $\xi$ & $\gamma_{\mathrm{L}}$ & $-\xi$ \\
\hline horizontal & $\leftarrow \cdot \rightarrow$ & $\frac{d_{0}}{2 \mu p s_{\mathrm{T}}^{2}}$ & $-\frac{\xi^{2}}{\gamma_{\mathrm{L}}}$ & $-\xi$ & $-\frac{\xi^{2}}{\gamma_{\mathrm{L}}}$ & $\xi$ \\
\hline \multicolumn{7}{|c|}{ concentrated single couples } \\
\hline vertical & & $\frac{m_{0}}{2 \mu p s_{\mathrm{T}}^{2}}$ & $\xi$ & $\frac{\xi^{2}}{\gamma_{\mathrm{T}}}$ & $-\xi$ & $\frac{\xi^{2}}{\gamma_{\mathrm{T}}}$ \\
\hline horizontal & $\stackrel{\uparrow}{\downarrow}$ & $\frac{m_{0}}{2 \mu p s_{\mathrm{T}}^{2}}$ & $-\xi$ & $\gamma_{\mathrm{T}}$ & $\xi$ & $\gamma_{\mathrm{T}}$ \\
\hline $\begin{array}{l}\text { double couple } \\
\text { without moment }\end{array}$ & $\vec{\downarrow}^{\prime} \leftarrow$ & $\frac{m_{0}}{\mu p s_{\mathrm{T}}^{2}}$ & $\xi$ & $-\frac{\gamma_{\mathrm{T}}^{2}-\xi^{2}}{\gamma_{\mathrm{T}}}$ & $-\xi$ & $-\frac{\gamma_{\mathrm{T}}^{2}-\xi^{2}}{\gamma_{\mathrm{T}}}$ \\
\hline centre of dilatation & $\stackrel{\uparrow}{\downarrow} \rightarrow$ & $\frac{\rho s_{\mathrm{L}}^{2}}{2 p} d_{0}$ & $\frac{1}{\gamma_{\mathrm{L}}}$ & 0 & $\frac{1}{\gamma_{\mathrm{L}}}$ & 0 \\
\hline centre of rotation & $\stackrel{\uparrow}{\downarrow}$ & $\frac{m_{0}}{2 \mu p}$ & 0 & $\frac{1}{\gamma_{\mathrm{T}}}$ & 0 & $\frac{1}{\gamma_{\mathrm{T}}}$ \\
\hline $\begin{array}{l}\text { edge dislocation } \\
\text { or vertical strike } \\
\text { fault }\end{array}$ & $\perp$ & $\frac{b_{0}}{p s_{\mathrm{T}}^{2}}$ & $\xi$ & $-\frac{\gamma_{\mathrm{T}}^{2}-\xi^{2}}{\gamma_{\mathrm{T}}}$ & $-\xi$ & $-\frac{\gamma_{\mathrm{T}}^{2}-\xi^{2}}{\gamma_{\mathrm{T}}}$ \\
\hline
\end{tabular}

${ }^{\mathrm{a}}$ The relations between phase-related source function and source function are

$$
\begin{array}{ll}
\phi_{0-}(\xi, p)=F(p) \phi_{0-}(\xi) \mathrm{e}^{+p \gamma_{\mathrm{L}} h_{s}}, & \psi_{0-}(\xi, p)=F(p) \psi_{0-}(\xi) \mathrm{e}^{+p \gamma_{\mathrm{T}} h_{s}}, \\
\phi_{0+}(\xi, p)=F(p) \phi_{0+}(\xi) \mathrm{e}^{-p \gamma_{\mathrm{L}} h_{s}}, & \psi_{0+}(\xi, p)=F(p) \psi_{0+}(\xi) \mathrm{e}^{-p \gamma_{\mathrm{T}} h_{s}} .
\end{array}
$$

${ }^{\mathrm{b}}$ All loadings are assumed with unit step time dependence $H(t)$ and with unit magnitude.

and $s^{*}$ is given by

$$
\boldsymbol{s}^{*}=\left(0,0, \ldots, \boldsymbol{s}_{-}^{(i)}\left(-h_{s_{i}}\right), \boldsymbol{s}_{+}^{(i)}\left(-h_{s_{i}}\right), \ldots, 0,0\right)^{\mathrm{T}} \text { for all } y .
$$

Note that $\boldsymbol{s}_{+}^{(i)}\left(-h_{s_{i}}\right)$ and $\boldsymbol{s}_{-}^{(i)}\left(-h_{s_{i}}\right)$ are determined by a body source at $y=-h_{s}$ in an infinite medium, and the material properties are the same as medium $(i)$. Several types of source function in an infinite domain are listed in table 1. For a more general type of loading conditions within the medium, the solution can be obtained by replacing the source vectors $\boldsymbol{s}_{0}^{*}$ and $\boldsymbol{s}^{*}$.

The analytic results for a layered medium in the transform domain are exact and can be expressed in a simple closed series form, with each term representing a physical transient wave. Each component of the matrix solution could then be identified with the solution derived by the theory of generalized rays. The solution 
in the time domain can be obtained by means of the inverse transform formulation in $(3.4 b)$, i.e.

$$
\boldsymbol{b}(x, y, t)=\frac{-p}{4 \pi^{2}} \int_{\xi_{1}-\infty \mathrm{i}}^{\xi_{1}+\infty \mathrm{i}} \mathrm{e}^{p \xi x} \int_{p_{1}-\infty \mathrm{i}}^{p_{1}+\infty \mathrm{i}} \hat{\boldsymbol{b}}(y ; \xi, p) \mathrm{e}^{p t} \mathrm{~d} p \mathrm{~d} \xi
$$

As an alternative, the transient solution in time domain can be accomplished by the application of Cagniard's method (Cagniard 1939). The idea behind Cagniard's method is to deform the path of integration in the complex $\xi$-plane in such a manner that the inverse Laplace transform along a new path of integration can be obtained by inspection and the convolution theorem. The method of Cagniard is surveyed in detail in the textbook written by Achenbach (1973) and in the review article by Pao \& Gajewski (1977). The numerical calculation of the exact transient solution based on Cagniard's method and the comparison between experimental waveform and theoretical results will be discussed in the second part of this paper.

\section{Conclusions}

A matrix method is proposed in this study to analyse transient waves in a multilayered medium subjected to in-plane dynamic loadings. With aids of the Bromwich matrix expansion, the solution in the transform domain is arranged into a power series of the reflection and transmission matrix $\boldsymbol{R}$. Each term of the power series represents a group of waves that are reflected by, or transmitted through, the interface the same number of times. The final solution in the transform domain is a series composed of the product of the receiver matrix $\boldsymbol{R}_{\boldsymbol{c} v}$, the power matrix of $\boldsymbol{R}$ and a source vector $\boldsymbol{s}$. The connection between the proposed method and the generalized ray method is established by considering the reflection and transmission of waves at a single interface.

For loadings applied in the interior of layers, the solution is obtained without introducing an artificial interface, only by replacing the source vector with a suitable one, as stated in $\S 6$. For a column of sources within the layered medium, the solution can be obtained easily by replacing the source vector $s$ correspondingly by the principle of superposition. Furthermore, if there is a column of receivers, the solution is readily obtained since the displacement potentials in each layer are given in the proposed solution.

The authors gratefully acknowledge the financial support of this research by the National Science Council (Republic of China) under grant NSC 87-2212-E-002-035.

\section{References}

Achenbach, J. D. 1973 Wave propagation in elastic solid. Amsterdam: North-Holland.

Boore, D. M., Aki, K. \& Todd, T. 1971 A two-dimensional moving dislocation model for a strike-slip fault. Bull. Seism. Soc. Am. 61, 177-194.

Cagniard, L. 1939 Reflexion et refraction des ondes seismiques progressives. Paris: CauthiersVillars. (Transl. Flinn, E. A. \& Dix, C. H. 1962 Reflection and refraction of progressive seismic waves. McGraw-Hill.)

Caviglia, G. \& Morro, A. 1994 Wave propagation in dissipative stratified layer. Wave Motion 19, 51-66. 
Ceranoglu, A. N. \& Pao, Y.-H. 1981 Propagation of elastic pulses and acoustic emission in a plate. ASME J. Appl. Mech. 48, 125-147.

Davids, N. 1959 Transient analysis of stress-wave penetration in plate. ASME J. Appl. Mech. 26, 651-660.

Ewing, W. M., Jardetzky, W. S. \& Press, F. 1957 Elastic waves in layered media. McGraw-Hill.

Fuchs, K. 1968 The reflection of spherical waves from transition zones with arbitrary depthdependent elastic moduli and density. J. Phys. Earth 16, 27-41.

Fuchs, K. \& Müller, G. 1971 Computation of synthetic seismograms with the reflectivity method and comparison with observations. Geophys. J. R. Astron. Soc. 23, 417-433.

Gilbert, F. \& Backus, G. E. 1966 Propagator matrices in elastic wave and vibration problems. Geophysics 31, 326-332.

Haskell, N. 1953 The dispersion of surface waves on multi-layered media. Bull. Seism. Soc. Am. 43, 17-34.

Kennett, B. L. N. 1983 Seismic wave propagation in stratified media. Cambridge University Press.

Kennett, B. L. N. \& Kerry, N. J. 1979 Seismic waves in a stratified half-space. Geophys. J. R. Astron. Soc. 44, 557-583.

Knopoff, L. 1958 Surface motion of a thick plate. J. Appl. Phys. 29, 661-670.

Kotulski, Z. 1990 Elastic waves in randomly stratified medium. Analytical results. Acta Mechanica 83, 61-75.

Lauriks, W., Allard, J. F., Depollier, C. \& Cops, A. 1991 In homogeneous plane waves in layered materials including fluid, solid and porous layers. Wave Motion 13, 329-336.

Ma, C. C. \& Huang, K. C. 1996 Analytical transient analysis of layered composite medium subjected to dynamic inplane impact loadings. Int. J. Solids Struct. 33, 4223-4238.

Mencher, A. G. 1953 Epicentral displacement caused by elastic waves in an infinite slab. J. Appl. Phys. 24, 1240-1246.

Müller, G. 1968a Theoretical seismograms for some types of point-source in layered media. I. Theory. Z. Geophys. B 34, 15-35.

Müller, G. $1968 b$ Theoretical seismograms for some types of point-source in layered media. II. Numerical calculations. Z. Geophys. B 34, 147-162.

Müller, G. 1969 Theoretical seismograms for some types of point-source in layered media. III. Single forces and dipole sources of arbitrary orientation. Z. Geophys. B 35, 347-371.

Müller, G. 1985 The reflectivity method: a tutorial. J. Geophys. 58, 153-174.

Nayfeh, A. H. 1991 The general problem of elastic wave propagation in multi-layered anisotropic media. J. Acoust. Soc. Am. 89, 1521-1531.

Nayfeh, A. H. 1995 Wave propagation in layered anisotropic media. Elsevier.

Pao, Y. H. \& Gajewski, R. 1977 The generalized ray theory and transient responses of layered elastic solids. In Physical acoustics, vol. 13, ch. 6, pp. 184-266 (ed. W. P. Mason \& R. N. Thurston). Academic.

Pekeris, C. L., Alterman, Z., Abramovici, F. \& Jarosh, H. 1965 Propagation of a compressional pulse in a layered solid. Rev. Geophys. 3, 25-47.

Peterson, B. \& Ström, S. 1974 Matrix formulation of acoustic scattering from an arbitrary number of scatterers. J. Acoust. Soc. Am. 56, 771-780.

Scott, J. F. M. 1985 The statistics of waves propagating in a one-dimensional random medium. Proc. R. Soc. Lond. A 398, 341-363.

Spencer, T. W. 1960 The method of generalized reflection and transmission coefficients. Geophysics 25, 625-641.

Thomson, W. T. 1950 Transmission of elastic waves through a stratified solid medium. J. Appl. Phys. 21, 89-93.

Ziegler, F. 1977 Wave propagaion in periodic and disordered layered composite elastic materials. Int. J. Solids Struct. 13, 293-305. 